\title{
OPTIMIZATION OF CRYSTAL GROWTH WITH DIFFUSION*
}

\author{
By \\ GUSTAF GRIPENBERG
}

Helsinki University of Technology, SF-02150 Espoo 15

\begin{abstract}
A crystal growth process involving diffusion in a half-space is studied. Both the diffusion coefficient and the interface reaction term are assumed to depend on a control parameter that is (e.g.) a function of temperature. The thickness of the deposited film after a given time or the time to reach a certain thickness is to be optimized. The full Stefan problem is not considered and the diffusion coefficient is assumed to vary slowly with the parameter.
\end{abstract}

1. Introduction and statement of results. The purpose of this paper is to find a function $\alpha$ with values in the interval $\left[\alpha_{1}, \alpha_{2}\right]$ such that if $\phi$ satisfies the diffusion equation

$$
\begin{gathered}
\partial \phi(\tau, x) / \partial \tau=D(\alpha(\tau)) \partial^{2} \phi(\tau, x) / \partial x^{2}, \quad x>0, \tau>0, \\
D(\alpha(\tau)) \partial \phi(\tau, x) /\left.\partial x\right|_{x=0}=A(\alpha(\tau), \quad \phi(\tau, 0)), \tau>0, \\
\phi(0, x)=\phi_{0}, x \geq 0, \lim _{x \rightarrow \infty} \phi(\tau, x)=\phi_{0}, \quad \tau \geq 0,
\end{gathered}
$$

then $C\left(\tau_{0}, \alpha\right) \stackrel{\text { def }}{=} \int_{0}^{t_{0}} D(\alpha(\tau)) \partial \phi(\tau, x) /\left.\partial x\right|_{x=0} d \tau$ is as large as possible for a given $\tau_{0}$ or $\tau_{0}$ is as small as possible and $C\left(\tau_{0}, \alpha\right) \geq \rho_{0}$ where $\rho_{0}$ is a given positive number. The equation (1.1) can be thought of as modeling the growth of a crystal from a solution where $\phi$ is the concentration of growth units, $D$ is the diffusion coefficient and the term $A$ describes the interface kinetics (see e.g. [1] and [8]). The parameter appearing in $D$ and $A$ can be a function of temperature and the reason for not always using the absolute temperature as a parameter in the formulation of the problem is that we assume that the functions $D$ and $A$ satisfy certain convexity properties that are unreasonable if the parameter used is directly proportional to the temperature. One could for example take $A(p, y)=p\left(\phi-k p^{\mu}\right)$ where $p=k_{1} \exp \left(-\mu_{1} / \theta\right), \theta$ is the absolute temperature, and $p$ represents the reaction constant and $k p^{\mu}$ the equilibrium concentration. The term $C\left(\tau_{0}, \alpha\right)$ is proportional to the thickness of the deposited film which is assumed to have such a large density, compared to the concentration in the solution, that the full Stefan problem can be neglected.

It is not essential that the diffusion take place on the whole half-line $0<x<\infty$. One could just as well consider the interval $0<x<L$ or the region outside a cylinder or a sphere (with appropriate symmetry assumptions) because the Green's functions for the boundary-value problems for the diffusion equation in these regions have exactly the same properties as the function $(\pi t)^{-1 / 2}$ that is used here (i.e. they are completely monotone and behave like $c t^{-1 / 2}$ as $t \rightarrow 0^{+}$).

\footnotetext{
* Received October 8, 1981.
} 
The main difficulties in the argument below are due to the fact that one allows the diffusion coefficient $D$ to depend on the control parameter, but it will be crucial for parts of the proofs that one assume that the derivative of $D$ with respect to the parameter is sufficiently small. There will be no restrictions on the control function $\alpha$ other than that it take values in the interval $\left[\alpha_{1}, \alpha_{2}\right]$, and this may of course also be somewhat unrealistic.

We first consider the problem of maximizing the thickness of the deposited film because the minimum-time problem can then be solved by almost the same argument.

Theorem 1. Assume that $\tau_{0}>0, \alpha_{1}<\alpha_{2}, \phi_{0}>0$ and that

$D: \quad\left[\alpha_{1}, \alpha_{2}\right] \rightarrow[1, \infty)$ is twice continuously differentiable, $1 / D$ is convex and either $D$ is a constant or $D^{\prime}$ is strictly positive on $\left[\alpha_{1}, \alpha_{2}\right]$,

$A: \quad\left[\alpha_{1}, \alpha_{2}\right] \times[0, \infty) \rightarrow(-\infty, \infty)$ is twice continuously differentiable and

$A(p, \cdot)$ is nondecreasing for each $p \in\left[\alpha_{1}, \alpha_{2}\right]$,

there exists a continuous nondecreasing function $\gamma:\left[\alpha_{1}, \alpha_{2}\right] \rightarrow[0, \infty)$ such that $A(p, y) \geq 0$ when $y \geq \gamma(p), A(p, y) \leq 0$ when $0 \leq y \leq \gamma(p)$, and $\gamma\left(\alpha_{1}\right) \leq$ $\phi_{0} \leq \gamma\left(\alpha_{2}\right)$,

$\partial^{2} A(p, y) / \partial y^{2}<0$ and $\partial^{2} B(p, y) / \partial y^{2}<0$ on $\left[\alpha_{1}, \alpha_{2}\right] \times\left[\gamma\left(\alpha_{1}\right), \gamma\left(\alpha_{2}\right)\right]$ where $B(p, y)=A(p, y) / D(p)$.

Then one can find a number $\delta>0$ such that

$$
0 \leq D^{\prime}(p) \leq \delta, \quad p \in\left[\alpha_{1}, \alpha_{2}\right],
$$

implies that there exists a unique (a.e.) function $\alpha_{*}:\left[0, \tau_{0}\right) \rightarrow\left[\alpha_{1}, \alpha_{2}\right]$ such that $C\left(\tau_{0}, \alpha_{*}\right) \geq C\left(\tau_{0}, \alpha\right)$ for all measurable functions $\alpha:\left[0, \tau_{0}\right] \rightarrow\left[\alpha_{1}, \alpha_{2}\right]$ where $C\left(\tau_{0}, \alpha\right)=$ $\int_{0}^{\tau_{0}} D(\alpha(\tau)) \partial \phi(\tau, x) /\left.\partial x\right|_{x=0} d \tau$ and $\phi$ is the solution of Eq. (1.1). Moreover, the function $\alpha_{*}$ is continuous and can be constructed through an iteration procedure.

The next result involves the problem of finding a function $\alpha$ such that $C\left(\tau_{0}, \alpha\right) \geq \rho_{0}$ and $\tau_{0}$ is as small as possible.

THEOREM 2. Let $\rho_{0}>0, \alpha_{1}<\alpha_{2}, \phi_{0}>0$ and assume that (1.2)-(1.6) hold. If $\delta>0$ is sufficiently small, then there exists a unique number $\tau_{0}^{*}$ and measurable function $\alpha^{*}$ : $\left[0, \tau_{0}^{*}\right] \rightarrow\left[\alpha_{1}, \alpha_{2}\right]$ such that $C\left(\tau_{0}^{*}, \alpha^{*}\right) \geq \rho_{0}$ and $\tau_{0}^{*} \leq \tau_{0}$ for each number $\tau_{0}$ and measurable function $\alpha:\left[0, \tau_{0}\right] \rightarrow\left[\alpha_{1}, \alpha_{2}\right]$ satisfying $C\left(\tau_{0}, \alpha\right) \geq \rho_{0}$. Moreover, the function $\alpha^{*}$ is continuous and can be found through an iteration procedure.

The proof of Theorem 2 relies on the fact that the optimal control function $\alpha^{*}$ of Theorem 2 is also the optimal control $\alpha_{*}$ for the problem in Theorem 1 when $\tau_{0}$ is taken to be $\tau_{0}^{*}$. To establish Theorems 1 and 2 one reduces the equation (1.1) to an integral equation using the appropriate Green's function. Then one uses some basic ideas from the theory of optimization of integral equations, and not the more specific results that can be found in the literature (see e.g. [6]).

For other (mainly asymptotic) results on solutions of diffusion equations with emphasis on the boundary terms, see e.g. [5] and [9] where the same approach of studying an integral equation is used.

2. Proof of Theorem 1. First we transform Eq. (1.1) into an integral equation. Let the function $\alpha$ be given on $[0, \infty)$ with values in the interval $\left[\alpha_{1}, \alpha_{2}\right]$ and define the function $\varphi$ 
by

$$
\varphi(\tau)=\int_{0}^{\tau} D(\alpha(s)) d s, \quad \tau \geq 0 .
$$

Since $D(\alpha(s)) \geq 1$ by (1.2), this function $\varphi$ has an inverse that we denote by $\psi$. It is straightforward to check, using (2.1), that the function $\phi$ satisfies (1.1) if and only if

$$
\begin{gathered}
\partial z(t, x) / \partial t=\partial^{2} z(t, x) / \partial x^{2}, \quad t>0, x>0, \\
D(\beta(t)) \partial z(t, x) /\left.\partial x\right|_{x=0}=A\left(\beta(t), \phi_{0}+z(t, 0)\right), \quad t>0, \\
z(0, x)=0, \quad x \geq 0, \quad \lim _{x \rightarrow \infty} z(t, x)=0, \quad t \geq 0,
\end{gathered}
$$

where $z(t, x)=\phi(\psi(t), x)-\phi_{0}$ and $\beta(t)=\alpha(\psi(t))$. If we take $u(t)=\partial z(t, x) /\left.\partial x\right|_{x=0}$ and

$$
a(t)=(\pi t)^{-1 / 2}, \quad t>0
$$

then (2.2) is equivalent to

$$
u(t)=B\left(\beta(t), \phi_{0}-\int_{0}^{t} a(t-s) u(s) d s\right), \quad t \geq 0
$$

(because $\left.z(t, x)=\int_{0}^{t}(\pi(t-s))^{-1 / 2} \exp \left(-x^{2}(4(t-s))^{-1}\right) u(s) d s\right)$. The quantity $C\left(\tau_{0}, \alpha\right)$ that is to be maximized becomes $\int_{0}^{T(\beta)} u(t) d t$ where $T(\beta)$ is the unique number satisfying

$$
\int_{0}^{T(\beta)} D(\beta(t))^{-1} d t=\tau_{0} .
$$

Once one has found the optimal function $\beta_{*}$ for this reformulated problem, one obtains the solution of the original one by taking $\alpha_{*}(\tau)=\beta_{*}(\varphi(\tau))$ and $\int_{0}^{\varphi(\tau)} D\left(\beta_{*}(t)\right)^{-1} d t=\tau$. The main difficulties are now of course due to the fact that the upper time limit $T(\beta)$ depends on $\beta$.

Before we prove that there exists an optimal control function $\beta_{*}$, we must derive some properties of the function

$$
v(t)=\phi_{0}-\int_{0}^{t} a(t-s) u(s) d s, \quad t \geq 0 .
$$

In view of (2.4) and (2.6) we conclude that the function $v$ satisfies the equation

$$
v(t)=\phi_{0}-\int_{0}^{t} a(t-s) B(\beta(s), v(s)) d s, \quad t \geq 0 .
$$

Below we denote the partial derivatives of e.g. $A$ and $B$ with respect to their first and second variables by the subindices 1 and 2 respectively.

LEMMA 1. If $\beta:[0, \infty) \rightarrow I \stackrel{\text { def }}{=}\left[\alpha_{1}, \alpha_{2}\right]$ is a given measurable function, then Eq. (2.7) has a unique continuous solution $v$ on $[0, \infty)$ that satisfies

$$
v(t) \in J \stackrel{\text { def }}{=}\left[\gamma\left(\alpha_{1}\right), \gamma\left(\alpha_{2}\right)\right], \quad t \geq 0 .
$$

Proof. Let $B(p, y)=B(p, 0)+B_{2}(p, 0) y, p \in I, y \leq 0$. It follows from standard results that Eq. (2.7) has a unique and continuous local solution. If we can prove that (2.8) holds for as long as the solution exists, then it is well known that we can extend the solution to the whole half-line. 
Assume that $t_{1}>0$ is such that we have a continuous solution of $(2.7)$ on $\left[0, t_{1}\right]$. Define $h(s)=B(\beta(s), v(s)) \cdot(v(s)-\gamma(\beta(s)))^{-1}$ so that $B(\beta(s), v(s))=h(s)(v(s)-\gamma(\beta(s)))$. By $(1.3)$ and (1.4) we see that the function $h$ is nonnegative and bounded on $\left[0, t_{1}\right]$. If we let $r(t, s)$ be the solution of the equation (cf. [4])

$$
\begin{aligned}
r(t, s)-a(t-s) h(s) & =\int_{s}^{t} r(t, u) a(u-s) h(s) d u \\
& =\int_{0}^{t} a(t-u) h(u) r(u, s) d u, \quad 0 \leq s \leq t \leq t_{1},
\end{aligned}
$$

then the solution $v$ of (2.7) can be written in the form

$$
v(t)=\phi_{0}\left(1-\int_{0}^{t} r(t, s) d s\right)+\int_{0}^{t} r(t, s) \gamma(\beta(s)) d s, \quad t \in\left[0, t_{1}\right] .
$$

Since $a$ is completely monotone and $h$ is nonnegative, it follows that $r(t, s) \geq 0$ and $\int_{0}^{t} r(t, s) d s \leq 1$ (use the results in [3, Thm. 1, Coroll. 1] or [7, Thm. 6] and an approximation argument that is needed since $h$ is not necessarily continuous). Hence we conclude from (2.9) and the fact that $\phi_{0} \in J$ that (2.8) holds on $\left[0, t_{1}\right]$, and the proof of Lemma 1 is completed.

Since Eq. (2.7) can be solved (for example by an iteration procedure), we can also solve Eq. (2.4). Let us denote the solution of (2.7) by $v=F(\beta)$ and that of (2.4) by $u=G(\beta)$. We proceed to show that there exists an optimal function $\beta$, but we do not yet show that it is unique.

Lemma 2. There exists at least one measurable function $\beta_{*}:[0, \infty) \rightarrow I$ such that if $u_{*}=$ $G\left(\beta_{*}\right), \beta$ is another measurable function: $[0, \infty) \rightarrow I$ and $u=G(\beta)$, then

$$
\int_{0}^{T\left(\beta_{*}\right)} u_{*}(t) d t \geq \int_{0}^{T(\beta)} u(t) d t
$$

Proof. Since Lemma 1 shows that $v$ is bounded independently of $\beta$, it follows that the solutions $u$ of (2.4) are uniformly bounded and therefore the numbers $\int_{0}^{T(\beta)} u(s) d s$ are bounded from above because we always have

$$
\tau_{0} D\left(\alpha_{1}\right)=T_{m} \leq T(\beta) \leq T_{M}=\tau_{0} D\left(\alpha_{2}\right) .
$$

We choose a sequence $\left\{\beta_{n}\right\}_{n=1}^{\infty}$ so that each function $\beta_{n}$ is measurable with its values in $I$ and

$$
\lim _{n \rightarrow \infty} \int_{0}^{T\left(\beta_{n}\right)} u_{n}(t) d t=\sup \left\{\int_{0}^{T(\beta)} u(t) d t \mid \beta(t) \in I, \quad u=G(\beta)\right\}
$$

where $u_{n}=G\left(\beta_{n}\right)$. From (2.3), (2.7) and (2.8) we deduce that the functions $v_{n}=F\left(\beta_{n}\right)$ are uniformly bounded and equicontinuous on $\left[0, T_{M}\right]$ and then we see from (2.4) and (2.6) that the functions $u_{n}$ are uniformly bounded too. This shows that we can choose a subsequence (denoted in the same way as the original one) so that there exist functions $\beta_{0}, w$ and $z$ and a number $T_{0}$ such that

$\beta_{n}$ converges weakly in $L^{2}\left(0, T_{M} ; R\right)$ towards $\beta_{0}$ and $u_{n}$ converges weakly in $L^{2}\left(0, T_{m} ; R\right)$ and weak-* in $L^{\infty}\left(0, T_{M} ; R\right)$ towards $w$ as $n \rightarrow \infty$ 
and

$v_{n} \rightarrow z$ uniformly on $\left[0, T_{M}\right]$ and $T\left(\beta_{n}\right) \rightarrow T_{0}$ as $n \rightarrow \infty$.

From (2.6), (2.12), (2.13) and the facts that $v_{n}=F\left(\beta_{n}\right), u_{n}=G\left(\beta_{n}\right)$ we see that

$$
z(t)=\phi_{0}-\int_{0}^{t} a(t-s) w(s) d s, t \in\left[0, T_{M}\right] .
$$

It is a consequence of $(2.12)$ that $\beta_{0}(t) \in I$ for a.e. $t \in\left[0, T_{M}\right]$. Moreover, it follows from (2.12) that there exist numbers $d_{j n} \geq 0$ such that $\sum_{j=n}^{N(n)} d_{j n}=1$ and $\sum_{j=n}^{N(n)} d_{j n} u_{j} \rightarrow w$ and $\sum_{j=n}^{N(n)} d_{j n} \beta_{j} \rightarrow \beta_{0}$ strongly in $L^{2}\left(0, T_{M} ; R\right)$ (see [10, Thm. 3.13]). Since we also have $\int_{0}^{T_{o}} D\left(\beta_{n}(t)\right)^{-1} d t \rightarrow \tau_{0}$ and $u_{n}-B\left(\beta_{n}(\cdot), z(\cdot)\right) \rightarrow 0$ uniformly on $\left[0, T_{M}\right]$ as $n \rightarrow \infty$ (see $(2.4)-(2.6),(2.12)$ and $(2.13))$, it follows from the concavity of the function $B(\cdot, y)$ and the convexity of the function $1 / D(\cdot)$ that

$$
w(t) \leq B\left(\beta_{0}(t), z(t)\right) \quad \text { a.e. } \quad t \in\left[0, T_{M}\right], \quad T\left(\beta_{0}\right) \geq T_{0} .
$$

By (2.11), (2.12) and (2.13) we know that

$$
\int_{0}^{T_{0}} w(t) d t=\sup \left\{\int_{0}^{T(\beta)} u(t) d t \mid \beta(t) \in I, \quad u=G(\beta)\right\}
$$

Since $v_{n}(t) \leq \gamma\left(\alpha_{2}\right)$ and $\beta_{n}(t) \leq \alpha_{2}$ it follows that $B\left(\alpha_{2}, v_{n}(t)\right) \leq B\left(\beta_{n}(t), v_{n}(t)\right)=u_{n}(t)$ because $B\left(\alpha_{2}, v_{n}(t)\right) \leq 0$ and if $B\left(\beta_{n}(t), v_{n}(t)\right) \leq 0$, then (1.4) and (1.5) imply that the function $B\left(\cdot, v_{n}(t)\right)$ is decreasing on $\left[\beta_{n}(t), \alpha_{2}\right]$. Hence (2.12) and (2.13) show that $w(t) \geq B\left(\alpha_{2}, z(t)\right)$, a.e. $t \in\left[0, T_{M}\right]$, and therefore we can by $(2.15)$ choose a measurable function $\beta_{*}$ so that

$$
\beta_{0}(t) \leq \beta_{*}(t) \leq \alpha_{2}, t \in\left[0, T_{0}\right]
$$

and

$$
w(t)=B\left(\beta_{*}(t), z(t)\right), \quad \text { a.e. } \quad t \in\left[0, T_{0}\right] .
$$

The last equality shows that if we take $\beta_{*}(t)=\alpha_{1}, t>T_{0}$ and $v_{*}=F\left(\beta_{*}\right), u_{*}=G\left(\beta_{*}\right)$, then $u_{*}(t)=w(t)$, a.e. on $\left[0, T_{0}\right]$. Since we also have $T\left(\beta_{*}\right) \geq T_{0}$ by (1.2), (2.15) and (2.17) and $u_{*}(t)=B\left(\alpha_{1}, v_{*}(t)\right) \geq 0$ when $t>T_{0}$ (see (1.4) and (2.8)), it follows from (2.16) that $\int_{0}^{T\left(\beta_{*}\right)} u_{*}(t) d t \geq \sup \left\{\int_{0}^{T(\beta)} u(t) d t \mid \beta(t) \in I, u=G(\beta)\right\}$. This completes the proof of Lemma 2.

There remains the problem of finding the optimal solution and we start by deriving an equation satisfied by $\beta_{*}$ and $v_{*}=F\left(\beta_{*}\right)$.

Lemma 3. If $\beta_{*}:[0, \infty) \rightarrow I$ is optimal in the sense of Lemma 2 and $v_{*}=F\left(\beta_{*}\right)$, then

$$
\begin{aligned}
q_{*}(t) B\left(\beta_{*}(t), v_{*}(t)\right)-\sup _{b \in I} A\left(b, v_{*}\left(T\left(\beta_{*}\right)\right)\right) / D\left(\beta_{*}(t)\right) \\
\quad=\sup _{p \in I}\left(q_{*}(t) B\left(p, v_{*}(t)\right)-\sup _{b \in I} A\left(b, v_{*}\left(T\left(\beta_{*}\right)\right)\right) / D(p)\right), \quad \text { a.e. } t \in\left[0, T\left(\beta_{*}\right)\right],
\end{aligned}
$$

where $q_{*}$ is the solution of the equation

$$
q_{*}(t)+\int_{t}^{T\left(\beta_{*}\right)} q_{*}(s) B_{2}\left(\beta_{*}(s), v_{*}(s)\right) a(s-t) d s=1, \quad t \in\left[0, T\left(\beta_{*}\right)\right] .
$$

Proof. To simplify the notation we write $V_{*}=v_{*}\left(T\left(\beta_{*}\right)\right)$ and $T_{*}=T\left(\beta_{*}\right)$. We may 
assume that $\beta_{*}(t)=p_{0}, t>T\left(\beta_{*}\right)$ where $p_{0} \in I$ is the number for which $A\left(p_{0}, V_{*}\right)=\sup _{p \in I}$ $A\left(p, V_{*}\right)$. We choose a measurable function $\eta$ which is zero outside a compact subset of $\left[0, T_{*}\right)$ and which is either nonnegative or nonpositive everywhere. Moreover, we assume that for sufficiently small $\varepsilon>0$ we have

$$
\beta(\varepsilon, t) \in I, t \geq 0 \quad \text { if } \beta(\varepsilon, t)=\beta_{*}(t)+\varepsilon \eta(t) .
$$

We take $v(\varepsilon, \cdot)=F(\beta(\varepsilon, \cdot)), u(\varepsilon, \cdot)=G(\beta(\varepsilon, \cdot))$ and $T(\varepsilon)=T(\beta(\varepsilon, \cdot))$.

Since $\beta_{*}$ is optimal and (2.20) holds we must of course have

$$
\limsup _{\varepsilon \rightarrow 0+} \varepsilon^{-1}\left(\int_{0}^{T(\varepsilon)} u(\varepsilon, t) d t-\int_{0}^{T_{*}} u_{*}(t) d t\right) \leq 0
$$

It is straightforward to check that $u(\varepsilon, t)$ is differentiable with respect to $\varepsilon$ and that the derivative at $\varepsilon=0$, which we denote by $w(t)$, satisfies the equation

$$
w(t)=B_{1}\left(\beta_{*}(t), v_{*}(t)\right) \eta(t)+B_{2}\left(\beta_{*}(t), v_{*}(t)\right)\left(-\int_{0}^{t} a(t-s) w(s) d s\right) .
$$

Using the fact that $q_{*}$ is the solution of (2.19), we easily check that

$$
\lim _{\varepsilon \rightarrow 0+} \varepsilon^{-1} \int_{0}^{T_{*}}\left(u(\varepsilon, t)-u_{*}(t)\right) d t=\int_{0}^{T_{*}} w(t) d t=\int_{0}^{T_{*}} q_{*}(t) B_{1}\left(\beta_{*}(t), v_{*}(t)\right) \eta(t) d t .
$$

We also have

$$
\lim _{\varepsilon \rightarrow 0+}\left(\varepsilon^{-1} \int_{T_{*}}^{T(\varepsilon)} u(\varepsilon, t) d t-\varepsilon^{-1} \int_{T_{*}}^{T(\varepsilon)} u_{*}(t) d t\right)=0
$$

From the definitions of $T(\varepsilon)$ and $T_{*}$ we obtain

$$
\int_{0}^{T_{*}}\left(D(\beta(\varepsilon, t))^{-1}-D\left(\beta_{*}(t)\right)^{-1}\right) d t+\int_{T_{*}}^{T(\varepsilon)} D(\beta(\varepsilon, t))^{-1} d t=0
$$

and therefore (recall that $\eta(t)=0$ in a neighborhood of $T_{*}$ )

$$
\int_{T_{*}}^{T(\varepsilon)} D\left(\beta_{*}(t)\right)^{-1} d t=\varepsilon \int_{0}^{T_{*}} D^{\prime}\left(\beta_{*}(t)\right) D\left(\beta_{*}(t)\right)^{-2} \eta(t) d t+o(\varepsilon) \quad \text { as } \quad \varepsilon \rightarrow 0^{+} .
$$

Suppose that $\eta(\cdot) \geq 0, \eta \not \equiv 0$. Then $T(\varepsilon)>T_{*}$ when $\varepsilon>0$ and since $\beta_{*}(t)=\beta(\varepsilon, t)=p_{0}$, $t>T_{*}$, it follows that $u_{*}$ has a right-hand limit at $T_{*}$; by (2.4), (2.6) and (2.24) we get

$$
\lim _{\varepsilon \rightarrow 0+} \varepsilon^{-1} \int_{T_{*}}^{T(\varepsilon)} u_{*}(t) d t=A\left(p_{0}, V_{*}\right) \int_{0}^{T_{*}} D^{\prime}\left(\beta_{*}(t)\right) D\left(\beta_{*}(t)\right)^{-2} \eta(t) d t .
$$

Combining this result with $(2.21)-(2.23)$, we have

$$
\int_{0}^{T_{*}}\left(q_{*}(t) B_{1}\left(\beta_{*}(t), v_{*}(t)\right)+A\left(p_{0}, V_{*}\right) D^{\prime}\left(\beta_{*}(t)\right) D\left(\beta_{*}(t)\right)^{-2}\right) \eta(t) d t \leq 0 .
$$

Next we assume that $\eta(\cdot) \leq 0$ and we pick a sequence $\left\{\varepsilon_{n}\right\}_{n=1}^{\infty}$ of positive numbers converging to 0 such that $\varepsilon_{n}^{-1} \int_{T_{*}}^{T\left(\varepsilon_{n}\right)} u_{*}(t) d t$ converges. By (2.4), (2.6) and (2.24) the limit 
must be of the form $A\left(p_{1}, V_{*}\right) \int_{0}^{T *} D^{\prime}\left(\beta_{*}(t)\right) D\left(\beta_{*}(t)\right)^{-2} \eta(t) d t$ for some $p_{1} \in I$. If $D^{\prime} \equiv 0$, then it does not matter what $p_{1}$ is, but otherwise we see that $p_{1}$ may depend on the sequence chosen but that it does not depend on $\eta$ in the sense that a change in $\eta$ can be compensated for by choosing another sequence $\left\{\varepsilon_{n}\right\}$. From $(2.21)-(2.23)$ we now get the inequality

$$
\int_{0}^{T_{*}}\left(q_{*}(t) B_{1}\left(\beta_{*}(t), v_{*}(t)\right)+A\left(p_{1}, V_{*}\right) D^{\prime}\left(\beta_{*}(t)\right) D\left(\beta_{*}(t)\right)^{-2}\right) \eta(t) d t \leq 0 .
$$

We must show that $p_{1}=p_{0}$. If $D^{\prime} \equiv 0$, then the value of $p_{1}$ is of no consequence and therefore we can assume that $D^{\prime}$ is strictly positive. If $\beta_{*}$ has a Lebesgue point $t_{0}$ in $\left(0, T_{*}\right)$ such that $\beta_{*}\left(t_{0}\right) \in \operatorname{int}(I)$, then we can find a nonnegative function $\eta_{0}$ not equal to 0 a.e. and such that we can take the function $\eta$ above to be both $+\eta_{0}$ and $-\eta_{0}$. Hence it follows from (2.25) and (2.26) that $p_{0}=p_{1}$ as the maximum of the function $A\left(\cdot, V_{*}\right)$ is achieved only at the point $p_{0}$ (see (1.5)). If such a Lebesgue point does not exist, then $\beta_{*}(t) \in\left\{\alpha_{1}, \alpha_{2}\right\}$ for a.e. $t \in\left[0, T_{*}\right]$.

Suppose first that $\beta_{*}(t)=\alpha_{2}$ for a.e. $t$ in a neighborhood of $T_{*}$ in $\left[0, T_{*}\right]$. In this case we have $p_{1}=\alpha_{2}$. Since the function $\eta$ can be chosen to approximate $\delta$-functionals, we conclude from (2.26) that

$$
q_{*}(t) B_{1}\left(\alpha_{2}, v_{*}(t)\right)+A\left(\alpha_{2}, V_{*}\right) D^{\prime}\left(\alpha_{2}\right) D\left(\alpha_{2}\right)^{-2} \geq 0
$$

for a.e. $t$ sufficiently close to $T_{*}$. If we let $t \rightarrow T_{*}-$ and recall the definitions of $B$ and $q_{*}$, then we get $A_{1}\left(\alpha_{2}, V_{*}\right) \geq 0$ and hence it follows from (1.5) and the definition of $p_{0}$ that $p_{1}=\alpha_{2}=p_{0}$. Next we consider the case when there is a sequence $\left\{t_{n}\right\}_{n=1}^{\infty}$ of Lebesgue points of $\beta_{*}$ converging to $T_{*}$ and $\beta_{*}\left(t_{n}\right)=\alpha_{1}$. From (2.25) and the fact that $\eta$ is arbitrary we obtain

$$
\left.q_{*}\left(t_{n}\right) B_{1}\left(\alpha_{1}, v_{*}\right)\left(t_{n}\right)\right)+A\left(p_{0}, V_{*}\right) D^{\prime}\left(\alpha_{1}\right) D\left(\alpha_{1}\right)^{-2} \leq 0
$$

and if we let $n \rightarrow \infty$, then we have

$$
A_{1}\left(\alpha_{1}, V_{*}\right) / D\left(\alpha_{1}\right) \leq\left(A\left(\alpha_{1}, V_{*}\right)-A\left(p_{0}, V_{*}\right)\right) D^{\prime}\left(\alpha_{1}\right) D\left(\alpha_{1}\right)^{-2} \leq 0
$$

by (1.2) and the definition of $p_{0}$. Therefore we are able to deduce from (1.5) that $\alpha_{1}=p_{0}$ and it remains to show that the assumption $p_{1}>\alpha_{1}$ leads to a contradiction. Let $\mu>0$ and define

$$
\begin{aligned}
\beta(\mu, t) & =\beta_{*}(t), & & t \in\left[0, T_{*}-\mu\right) \\
& =\alpha_{1}, & & t \geq T_{*}-\mu
\end{aligned}
$$

and take $T(\mu)=T(\beta(\mu, \cdot)), v(\mu, \cdot)=F(\beta(\mu, \cdot))$ and $u(\mu, \cdot)=G(\beta(\mu, \cdot))$. Since $\beta_{*}$ is optimal and $u(\mu, t)=u_{*}(t)$ when $t<T_{*}-\mu$, it follows that

Obviously we have

$$
\int_{T_{*}-\mu}^{T_{*}} u_{*}(t) d t \geq \int_{T_{*}-\mu}^{T(\mu)} u(\mu, t) d t
$$

$$
u(\mu, t)=A\left(\alpha_{1}, V_{*}\right) / D\left(\alpha_{1}\right)+o(1) \quad \text { as } \quad \mu \rightarrow 0+, \quad \text { uniformly for } \mathrm{t} \in\left[T_{*}-\mu, T(\mu)\right],
$$

and since it follows from the definitions of $T(\mu)$ and $T_{*}$ that

$$
\int_{T_{*}-\mu}^{T_{*}} D\left(\beta_{*}(t)\right)^{-1} d t=\int_{T_{*}-\mu}^{T(\mu)} D\left(\alpha_{1}\right)^{-1} d t=\left(T(\mu)-T_{*}+\mu\right) / D\left(\alpha_{1}\right)
$$


we conclude that

$$
\begin{aligned}
\int_{T_{*}-\mu}^{T_{*}}\left(A\left(\beta_{*}(t), v_{*}(t)\right)\right. & \left.-A\left(p_{1}, V_{*}\right)\right) D\left(\beta_{*}(t)\right)^{-1} d t \\
= & \int_{T_{*}-\mu}^{T_{*}} u_{*}(t) d t-A\left(p_{1}, V_{*}\right) D\left(\alpha_{1}\right)^{-1}\left(T(\mu)-T_{*}+\mu\right) \\
= & \int_{T_{*}-\mu}^{T_{*}} u_{*}(t) d t-\int_{T_{*}-\mu}^{T(\mu)} u(\mu, t) d t+o(1)\left(T(\mu)-T_{*}+\mu\right) \\
& +\left(A\left(\alpha_{1}, V_{*}\right)-A\left(p_{1}, V_{*}\right)\right) D\left(\alpha_{1}\right)^{-1}\left(T(\mu)-T_{*}+\mu\right) .
\end{aligned}
$$

This equality gives a contradiction when we divide it by $\mu$; let $\mu \rightarrow 0$ and use (2.27), (2.28), the definition of $p_{1}$ and the fact that $p_{1}>p_{0}=\alpha$, implies, by (1.5), that $A\left(\alpha_{1}, V_{*}\right)>$ $A\left(p_{1}, V_{*}\right)$. Thus we have shown that $p_{1}=p_{0}$ and the relation (2.18) follows from (1.2), (1.5), (2.25) and (2.26). This completes the proof of Lemma 3.

We proceed to show how one can find the optimal function $\beta_{*}$ by solving Eqs. (2.18) and (2.19). Since we will use the Banach fixed-point theorem we get uniqueness as a side result, but we must assume that $D^{\prime}$ is sufficiently small and this hypothesis has not been used so far. First we establish some auxiliary results.

Lemma 4. For each $c>0$, there exists a constant $Q$ such that if $T_{m} \leq T \leq T_{M}$, $h:[0, T] \rightarrow[0, c]$ is measurable and $z$ is the solution of the equation

$$
z(t)+\int_{t}^{T} z(s) h(s) a(s-t) d s=1, \quad t \in[0, T],
$$

then

$$
Q \leq z(t) \leq 1, \quad t \in[0, T] .
$$

Proof. Let $R$ be the solution of the equation

$$
R(t)+c \int_{0}^{t} R(t-s) a(s) d s=1, t \geq 0 .
$$

The fact that $a$ is locally integrable, positive and nonincreasing with $\log (a)$ convex implies that $R$ is nonincreasing and positive on $[0, \infty)$ (see [2]). We are going to show that we can take $Q=R\left(T_{M}\right)$ or equivalently that $w(t) \stackrel{\text { def }}{=} z(t)-R(T-t)$ is nonnegative. By (2.29) and (2.31) we know that $w$ satisfies the equation

$$
w(t)+\int_{t}^{T} w(s) h(s) a(s-t) d s=\int_{t}^{T} R(T-s)(c-h(s)) a(s-t) d s, \quad t \in[0, T] .
$$

Let $p(t, s)$ denote the solution of the equation

$$
p(t, s)-a(t-s)=\int_{s}^{t} a(t-u) h(u) p(u, s) d u=\int_{s}^{t} p(t, u) h(u) a(u-s) d u, \quad 0 \leq s \leq t \leq T .
$$

To show that such a solution exists one can use the argument in [4]. It follows essentially from $[3$, Th. 1] that $p(t, s) \geq 0$ because the results established there imply that $h(t) p(t, s)>0$ provided that $h$ is continuous and positive and one can then use an approximation argument to derive the result needed here. The solution $w$ of the equation (2.32) can be expressed 
in the form

$$
w(t)=\int_{t}^{T} R(T-s)(c-h(s)) p(s, t) d s
$$

and since $p(s, t) \geq 0$ and $h(s) \leq c$ we get $w(t) \geq 0$ and hence the first inequality in (2.30) holds with $Q=R\left(T_{M}\right)$. The second inequality follows then from (2.29). This completes the proof of Lemma 4.

The next auxiliary result involves the relation (2.18). Take the number $c$ in Lemma 4 to be $\sup _{p \in I, y \in J}\left|B_{2}(p, y)\right|$ and define

$$
\begin{aligned}
& P=P(y, z, \omega), y, z \in J, \omega \in[Q, 1] \text { if and only if } P \in I \text { and } \omega B(P, y) \\
& -\sup _{b \in I} A(b, z) / D(P)=\sup _{p \in I}\left(\omega B(p, y)-\sup _{b \in I} A(b, z) / D(p)\right) .
\end{aligned}
$$

From (1.2) and (1.5) we see that $P$ is a well-defined function since $\sup _{b \in I} A(b, z) \geq$ $A\left(\alpha_{1}, z\right) \geq 0$. We prove the following lemma.

Lemma 5. The function $P: J \times J \times[Q, 1] \rightarrow I$ defined in (2.33) is uniformly Lipschitzcontinuous in each variable and the Lipschitz constants with respect to the last two variables are bounded by $c_{1} \sup _{p \in I} D^{\prime}(p)$ where $c_{1}$ is a certain constant.

Proof. Let $P_{0}$ be the function defined in exactly the same manner as $P$, except that we do not make the restriction that the supremum be taken over the interval $I$. We may extend $A$ and $D$ in a suitable manner so that we always have a unique maximum and the concavity assumptions in (1.2) and (1.5) hold on the interval where the maximum will be found. It is clear that we must have

$$
P(y, z, \omega)=\max \left\{\alpha_{1}, \min \left\{\alpha_{2}, P_{0}(y, z, \omega)\right\}\right\} .
$$

Therefore it is sufficient to show that $P_{0}$ is continuously differentiable with respect to all of its three variables and that the absolute value of the derivative with respect to the last two variables are bounded by $c_{1} \sup _{p \in I} D^{\prime}(p)$ in the case when $P_{0}(z, y, \omega) \in I$. It is an obvious consequence of (1.3) and (1.5) that the function $\sup _{b \in I} A(b, z)$ is continuously differentiable with respect to $z$ (use the implicit function theorem and observe that if the supremum is achieved at $b(z)$ then $A_{1}(b(z), z) b^{\prime}(z)=0$ because either $A_{1}(b(z), z)=0$ or $\left.b^{\prime}(z)=0\right)$. The number $P_{0}(y, z, \omega)$ is determined as the solution of the equation

$$
\omega B_{1}(p, y)+\sup _{b \in I} A(b, z) D^{\prime}(p) D(p)^{-2}=0
$$

and hence we obtain the desired conclusion with the aid of (1.2), (1.3), (1.5) and the implicit function theorem (here it is essential that $1 \geq \omega \geq Q>0$ ). This completes the proof of Lemma 5.

Fix $T \in\left[T_{m}, T_{M}\right]$. We intend to solve the system of equations

$$
q(t)+\int_{t}^{T} q(s) B_{2}(P(v(s), v(T), q(s)), v(s)) a(s-t) d s=1, \quad t \in[0, T]
$$

and

$$
v(t)=\phi_{0}-\int_{0}^{t} a(t-s) B(P(v(s), v(T), q(s)), v(s)) d s, \quad t \in[0, T] .
$$

These equations are of course the same as (2.7) and (2.19) when we take 
$\beta(t)=P(v(t), v(T), q(t))$, as we should according to (2.18) and (2.33). But observe that we have not yet chosen $T$ to be $T(\beta)$. We can define a function $H_{T}: C([0, T] ; J) \rightarrow$ $C([0, T] ; J)$ in the following way: given a continuous function $v$ on $[0, T]$ with values in the interval $J$, we can find the solution $q$ of Eq. (2.34) (e.g. by iteration), and with the aid of Lemma 4 one can show that $q(t) \in[Q, 1]$. (Observe that it does not matter that now the function $h$ of Lemma 4 depends on $z$ as long as $0 \leq h(t) \leq c$.) If this function $q$ and the number $v(T)$ are substituted into Eq. (2.35), then one can (again using iteration) solve that equation and the solution is $H_{T}(v)$. From Lemma 1 one sees that we actually have $H_{T}(v) \in$ $C([0, T] ; J)$. We need the following result.

LEMMA 6. If the number $\delta$ that appears in (1.6) is sufficiently small, then the mapping $H_{T}$ is a contraction for each $T \in\left[T_{m}, T_{M}\right]$. If $v_{T}$ denotes the unique fixed point of $H_{T}$ and $q_{T}$ is the corresponding solution of (2.34), then $v_{T}$ and $q_{T}$ are absolutely continuous and there exist constants $c_{2}$ and $c_{3}$ (independent of $T$ ) such that

$$
\begin{gathered}
\left|v_{T}^{\prime}(t)\right| \leq c_{2}\left(t^{-1 / 2}+(T-t)^{-1 / 2}\right), \\
\left|q_{T}^{\prime}(t)\right| \leq c_{3}\left(t^{-1 / 2}+(T-t)^{-1 / 2}\right), \quad \text { a.e. } t \in(0, T) .
\end{gathered}
$$

Proof. The fact that $H$ is a contraction (provided that $\delta$ is sufficiently small) follows from (1.3) and Lemma 5 because it is straightforward to show that the mapping $v \rightarrow q$ defined by (2.34) is Lipschitz-continuous (but the Lipschitz constant need not be small) and that the mapping $(q, v(T)) \rightarrow H_{T}(v)$ is Lipschitz-continuous with a constant of the form $c_{4} \delta$. To prove these results one uses standard arguments that appear in a slightly different form in the second part of this proof.

Suppose next that we know that the function $v$ is absolutely continuous when we solve $q$ from (2.34). Then we see that $q$ is absolutely continuous too, and we have in fact

$$
\begin{aligned}
q^{\prime}(t) & +B_{2}(P(v(T), v(T), q(T)), v(T)) a(T-t)+\int_{t}^{T}\left(q^{\prime}(s) B_{2}(P(v(s), v(T), q(s)), v(s))\right. \\
& +q(s) B_{21}\left(P ( v ( s ) , v ( T ) , q ( s ) , v ( s ) ) \left(P_{1}\left(v(s), v(T), q(s) v^{\prime}(s)+P_{3}(v(s), v(T), q(s)) q^{\prime}(s)\right)\right.\right. \\
& \left.+q(s) B_{22}(P(v(s), v(T), q(s)), v(s)) v^{\prime}(s)\right) a(s-t) d s=0, \quad \text { a.e. } \quad t \in(0, T) .
\end{aligned}
$$

To simplify the notation we choose the constant $c_{5}$ to be such that (2.37), and (2.44) hold and the first one of these relations is

$$
\sup _{p \in I, y \in J}\left|B_{2}(p, y)\right|+\sup _{p \in I, y \in J}\left|B_{21}(p, y)\right| c_{6}+\sup _{p \in I, y \in J}\left|B_{22}(p, y)\right| \leq c_{5}
$$

where $c_{6}$ is an upper bound for the Lipschitz constants of $P$ with respect to each of its variables. If we take $r$ to be the solution of the equation

$$
r(t)=c_{5} a(t)+c_{5} \int_{0}^{t} a(t-s) r(s) d s, \quad t>0,
$$

then it follows from $(2.36)-(2.38)$ and the fact that $a(\cdot) \geq 0$ that

$$
\left|q^{\prime}(t)\right| \leq r(T-t)+\int_{t}^{T}\left|v^{\prime}(s)\right| r(s-t) d s, \quad \text { a.e. } \quad t \in[0, T) .
$$


Using (2.3) and (2.38) we can find a constant $c_{7}$ (depending on $T_{M}$ ) such that

$$
c_{7}^{-1} t^{-1 / 2} \leq r(t) \leq c_{7} t^{-1 / 2}, \quad t \in\left(0, T_{M}\right)
$$

If there now exists a constant $c_{8}$ such that

$$
\left|v^{\prime}(t)\right| \leq c_{8}\left(t^{-1 / 2}+(T-t)^{-1 / 2}\right), \quad \text { a.e. } \quad t \in(0, T)
$$

then it is easy to see from (2.39)-(2.41) that there also exists a constant $c_{9}$ (independent of $c_{8}$ ) such that

$$
\left|q^{\prime}(t)\right| \leq\left(c_{8}+1\right) c_{9}\left(t^{-1 / 2}+(T-t)^{-1 / 2}\right), \quad \text { a.e. } t \in(0, T) .
$$

If we know that $q$ is absolutely continuous when we solve $H_{T}(v) \stackrel{\text { def }}{=} w$ from (2.35), then we deduce that $w$ is absolutely continuous too and we have

$$
\begin{aligned}
w^{\prime}(t) & +B(P(\omega(0), v(T), q(0)), w(0)) a(t) \\
& +\int_{0}^{t} a(t-s)\left(B _ { 1 } ( P ( w ( s ) , v ( T ) , q ( s ) ) , w ( s ) ) \left(P_{1}(w(s), v(T), q(s)) w^{\prime}(s)\right.\right. \\
& \left.+P_{3}(w(s), v(T), q(s)) q^{\prime}(s)\right) \\
& \left.+B_{2}(P(w(s), v(T), q(s)), w(s)) w^{\prime}(s)\right) d s, \quad \text { a.e. } t \in(0, T) .
\end{aligned}
$$

We have assumed that

$$
\sup _{p \in I, y \in J}|B(p, y)|+\sup _{p \in I, y \in J}\left|B_{1}(p, y)\right|\left(c_{1}+c_{6}\right)+\sup _{p \in I, y \in J}\left|B_{2}(p, q)\right| \leq c_{5},
$$

and therefore it follows from Lemma 5, (2.38) and (2.43) that

$$
\left|w^{\prime}(t)\right| \leq r(t)+\int_{0}^{t} r(t-s) \delta\left|q^{\prime}(s)\right| d s, \quad \text { a.e. } t \in(0, T) .
$$

If we assume that (2.42) holds, then it is a consequence of this inequality and (2.40) that there exists a constant $c_{10}$ such that

$$
\left|w^{\prime}(t)\right| \leq c_{10}\left(1+\delta\left(c_{8}+1\right) c_{9}\right)\left(t^{-1 / 2}+(T-t)^{-1 / 2}\right), \quad \text { a.e. } t \in(0, t) .
$$

Since the fixed point $v_{T}$ is found by iteration, we conclude from (2.41) and (2.45) that if $\delta c_{9} c_{10}<1$, then every function in the iteration sequence is absolutely continuous and satisfies an inequality of the form (2.41) with $\left.c_{8}=\left(c_{10}+1\right)\left(1-\delta c_{9} c_{10}\right)^{-1}\right)$, and therefore the same is true for the limit function $v_{T}$. The second part of the claim (2.36) follows from (2.42). This completes the proof of Lemma 6.

Next we consider the following mapping: given $T \in\left[T_{m}, T_{M}\right]$, we can by Lemma 6 find the unique solution $v_{T}$ and $q_{T}$ of the system (2.34), (2.35) and extend these functions as $v_{T}(t)=v_{T}(T), q_{T}(t)=q_{T}(T), t>T$. We take $\beta_{T}(t)=P\left(v_{T}(t), v_{T}(T), q_{T}(t)\right)$ and then we determine $K(T)$ from the condition $\int_{0}^{K(T)} D\left(\beta_{T}(t)\right)^{-1} d t=\tau_{0}$. Thus $K(T)$ is a well-defined number in the interval $\left[T_{m}, T_{M}\right]$. If we can show that $K$ is a contraction, then by the Banach fixed-point theorem it has a unique fixed point $T_{0}$ and one deduces from Lemmas 2, 3, 5 and 6 and (2.33)-(2.35) that $T_{0}=T\left(\beta_{*}\right), \beta_{T_{0}}=\beta_{*}, v_{T_{0}}=v_{*}, q_{T_{0}}=q_{*}$ and that the optimal function $\beta_{*}$ is unique and continuous. Thus it remains to establish 
Lemma 7. The mapping $K:\left[T_{m}, T_{M}\right] \rightarrow\left[T_{m}, T_{M}\right]$ is a contraction, provided that $\delta$ is sufficiently small.

Proof. Choose two arbitrary numbers $T_{1}$ and $T_{2}$ in $\left[T_{m}, T_{M}\right]$ and assume that $T_{m}>k \stackrel{\text { def }}{=}$ $T_{2}-T_{1}>0$. We take

$$
\begin{aligned}
& v_{1}(t)=v_{T_{1}}(t), \quad v_{2}(t)=v_{T_{2}}(t+k), \\
& q_{1}(t)=q_{T_{1}}(t), \quad q_{2}(t)=q_{T_{2}}(t+k), \quad t \in\left[0, T_{1}\right] .
\end{aligned}
$$

From (2.34) and (2.46) we have

$$
\begin{aligned}
q_{1}(t)-q_{2}(t)= & -\int_{t}^{T_{1}}\left(q_{1}(s) B_{2}\left(P\left(v_{1}(s), v_{1}\left(T_{1}\right), q_{1}(s)\right), v_{1}(s)\right)\right. \\
& \left.-q_{2}(s) B_{2}\left(P\left(v_{2}(s), v_{2}\left(T_{1}\right), q_{2}(s)\right), v_{2}(s)\right)\right) a(s-t) d s, \quad t \in\left[0, T_{1}\right] .
\end{aligned}
$$

Using (2.37), (2.38) and the fact that $q_{j}(t) \in[Q, 1], v_{j}(t) \in J, t \in\left[0, T_{1}\right]$, we conclude from (2.47) that

$$
\left|q_{1}(t)-q_{2}(t)\right| \leq \int_{t}^{T_{1}}\left(\left|v_{1}(s)-v_{2}(s)\right|+\left|v_{1}\left(T_{1}\right)-v_{2}\left(T_{1}\right)\right|\right) r(s-t) d s, \quad t \in\left[0, T_{1}\right] .
$$

From (2.35) and (2.46) we obtain

$$
\begin{aligned}
v_{1}(t)-v_{2}(t)= & -\int_{0}^{t} a(t-s)\left(B\left(P\left(v_{1}(s), v_{1}\left(T_{1}\right), q_{1}(s)\right), v_{1}(s)\right)\right. \\
& \left.-B\left(P\left(v_{2}(s), v_{2}\left(T_{1}\right) q_{2}(s)\right), v_{2}(s)\right)\right) d s \\
& +\int_{0}^{k} a(t+k-s) B\left(P\left(v_{T_{2}}\left(T_{2}\right), q_{T_{2}}(s)\right), v_{T_{2}}(s)\right) d s, \quad t \in\left[0, T_{1}\right] .
\end{aligned}
$$

By Lemma 5, (2.38), (2.44), (2.49) and the fact that $a$ is nonnegative it follows that

$$
\begin{aligned}
\left|v_{1}(t)-v_{2}(t)\right| \leq \int_{t}^{t+k} r(s) d s+\int_{0}^{t} r(t-s)\left(\delta\left|q_{1}(s)-q_{2}(s)\right|\right. \\
\\
\left.+\delta\left|v_{1}\left(T_{1}\right)-v_{2}\left(T_{1}\right)\right|\right) d s, \quad t \in\left[0, T_{1}\right] .
\end{aligned}
$$

If we insert (2.49) into this inequality, then we get

$$
\begin{aligned}
\left|v_{1}(t)-v_{2}(t)\right| \leq & \int_{t}^{t+k} r(s) d s+\delta \int_{0}^{T_{1}} k(t, s)\left|v_{1}(s)-v_{2}(s)\right| d s \\
& +\delta\left(\int_{0}^{t} r(s) d s+\int_{0}^{T_{1}} k(t, s) d s\right)\left|v_{1}\left(T_{1}\right)-v_{2}\left(T_{1}\right)\right|, \quad t \in\left[0, T_{1}\right]
\end{aligned}
$$

where

$$
k(t, s)=\int_{0}^{\min \{t, s\}} r(t-u) r(s-u) d u, \quad t, s>0 .
$$

Using $(2.40)$ we deduce that there exists a constant $c_{11}$ such that

$$
0 \leq k(t, s) \leq c_{11}|t-s|^{-1 / 2}, \quad t, s>0 .
$$


If $\delta$ is so small that $2 \delta\left(\int_{0}^{t} r(s) d s+\int_{0}^{T_{M}} k(t, s) d s\right)<1, t \in\left[0, T_{M}\right]$, then it follows from (2.50) that

$$
\left|v_{1}\left(T_{1}\right)-v_{2}\left(T_{1}\right)\right| \leq 2 \int_{T_{1}}^{T_{1}-k} r(s) d s+2 \delta \int_{0}^{T_{1}} k\left(T_{1}, s\right)\left|v_{1}(s)-v_{2}(s)\right| d s
$$

so that we also have by $(2.50)$

$$
\begin{aligned}
\left|v_{1}(t)-v_{2}(t)\right| \leq & \int_{t}^{t+k} r(s) d s+2 \int_{T_{1}}^{T_{1}+k} r(s) d s \\
& +\delta \int_{0}^{T_{1}}\left(k(t, s)+2 k\left(T_{1}, s\right)\right)\left|v_{1}(s)-v_{2}(s)\right| d s
\end{aligned}
$$

If we require that $2 \delta \int_{0}^{T_{M}}\left(k(t, s)+2 k\left(T_{1}, s\right)\right) d s \leq 1$ and $2 \delta \int_{0}^{T_{M}}\left(k(t, s)+2 k\left(T_{1}, s\right)\right)\left(\int_{s}^{s+k} r(u) d u\right)$ $d s \leq \int_{t}^{t+k} r(u) d u, t \in\left[0, T_{M}\right], T_{1} \in\left[0, T_{M}\right]$ (this is possible by (2.40) and (2.51)), then we can deduce from (2.52) that

$$
\left|v_{1}(t)-v_{2}(t)\right| \leq 2 \int_{t}^{t+k} r(s) d s+4 \int_{T_{1}}^{T_{1}+k} r(s) d s, \quad t \in\left[0, T_{1}\right] .
$$

(Take $w(t)=\left|v_{1}(t)-v_{2}(t)\right|-2 \int_{t}^{t+k} r(s) d s$ and find the supremum of this function on $\left[0, T_{1}\right]$.) But then it follows from (2.40) and (2.53) that

$$
\left|v_{1}(t)-v_{2}(t)\right| \leq 6 c_{7} t^{-1 / 2} k, \quad t \in\left[0, T_{1}\right] .
$$

If we insert this inequality into (2.48) and use (2.40) then we find a constant $c_{12}$ such that

$$
\left|q_{1}(t)-q_{2}(t)\right| \leq c_{12} t^{-1 / 2} k, t \in\left[0, T_{1}\right] .
$$

From (2.36), (2.46), (2.54), (2.55) and the way in which the functions $v_{T}$ and $q_{T}$ were extended we conclude that there exists a constant $c_{13}$ such that

$$
\begin{aligned}
& \left|v_{T_{1}}(t)-v_{T_{2}}(t)\right|+\left|q_{T_{1}}(t)-q_{T_{2}}(t)\right| \leq c_{13}\left(t^{-1 / 2}+\left(T_{1}-t\right)^{-1 / 2}\right) k, \quad t \in\left(0, T_{1}\right), \\
& \left|v_{T_{1}}(t)-v_{T_{2}}(t)\right|+\left|q_{T_{1}}(t)-q_{T_{2}}(t)\right| \leq c_{13}\left(k+\left(T_{2}-t\right)^{1 / 2}\right), \quad t \in\left[T_{1}, T_{2}\right], \\
& \left|v_{T_{1}}(t)-v_{T_{2}}(t)\right|+\left|q_{T_{1}}(t)-q_{T_{2}}(t)\right| \leq c_{13} k, \quad t>T_{2} .
\end{aligned}
$$

If we moreover recall that $v_{T_{1}}\left(T_{1}\right)-v_{T_{2}}\left(T_{2}\right)=v_{T_{1}}(t)-v_{T_{2}}(t), t>T_{2}$, then it follows from Lemma $5,(1.2),(1.6),(2.55)$ and the definition of the function $\beta_{T}$ that there exists a constant $c_{14}$ such that

$$
\int_{0}^{T_{M}}\left|D\left(\beta_{T_{1}}(t)\right)^{-1}-D\left(\beta_{T_{2}}(t)\right)^{-1}\right| d t \leq c_{14} \delta k
$$

This inequality combined with the definition of the function $K$ implies that

$$
\left|K\left(T_{1}\right)-K\left(T_{2}\right)\right| \leq D\left(\alpha_{2}\right) c_{14} \delta\left|T_{2}-T_{1}\right|
$$

and thus we get the desired assertion. This completes the proof of Lemma 7.

3. Proof of Theorem 2. We use the same notation as in the proof of Theorem 1 and we observe that the reduction of the problem to the integral equation form can still be done. Thus we have to find a function $\beta$ such that if $u$ satisfies (2.4) and $\int_{0}^{T} u(t) d t \geq \rho_{0}$ for some $T$, then $\int_{0}^{T} D(\beta(t))^{-1} d t$ is as small as possible. 
We let $\tau(\beta)=\int_{0}^{T} D(\beta(t))^{-1} d t$ where $T$ is the smallest number such that $\int_{0}^{T} u(t) d t \geq \rho_{0}$ and $u=G(\beta)$. Obviously there exist positive numbers $\tau_{m}$ and $\tau_{M}$ such that if there exists an optimal solution to the problem at hand, then the corresponding number $\tau(\beta)$ must lie in the interval $\left[\tau_{m}, \tau_{M}\right]$. For each $\tau_{0} \in\left[\tau_{m}, \tau_{M}\right]$ there exists a unique optimal solution of the problem considered in Theorem 1 that we denote by $\beta_{\tau_{0}}$. (It is easy to check that our choice of $\delta$ in the proof of Theorem 1 can be made uniform for $\tau_{0} \in\left[\tau_{m}, \tau_{M}\right]$, because a change in $\tau_{0}$ will only change $T_{m}$ and $T_{M}-$ see (2.10).) We claim that

$$
\int_{0}^{T\left(\beta_{\tau}\right)} u_{\tau}(t) d t \text { is a nondecreasing continuous function of } \tau \text {, }
$$

where $T\left(\beta_{\tau}\right)$ is defined in (2.5) (with $\tau_{0}$ replaced by $\tau$ ) and $u_{\tau}=G\left(\beta_{\tau}\right)$. Assume that (3.1) does not hold and that there exist numbers $\tau_{2}>\tau_{1}$ in $\left[\tau_{m}, \tau_{M}\right]$ such that

$$
\int_{0}^{T\left(\beta_{\tau, 1}\right)} u_{\tau_{1}}(t) d t>\int_{0}^{T\left(\beta_{\tau, 2}\right)} u_{\tau_{2}}(t) d t .
$$

Since $\beta_{\tau_{2}}$ is optimal we get a contradiction by taking $\beta(t)=\beta_{\tau_{1}}(t), t \in\left[0, T\left(\beta_{\tau_{1}}\right)\right]$ and $\beta(t)=$ $\alpha_{1}, t>T\left(\beta_{\tau_{1}}\right)$ so that if $u=G(\beta)$, then $u(t)=u_{\tau_{1}}(t), t \in\left[0, T\left(\beta_{\tau_{1}}\right)\right]$ and $u(t) \geq 0$ otherwise. The continuity of the function appearing in (3.1) is established by a similar argument that uses the fact that the functions $u=G(\beta)$ are bounded independently of $\beta$.

Since (3.1) holds we can find the smallest number $\tau^{*} \in\left[\tau_{m}, \tau_{M}\right]$ such that $\int_{0}^{T\left(\beta_{\tau *}\right)} u_{\tau *}(t)$ $d t=\rho_{0}$. We define $\beta^{*}=\beta_{\tau *}$ and we claim that $\beta^{*}$ is the desired optimal control. Suppose that this is not the case, but that there exists another measurable function $\beta$ taking its values in the interval $I$ such that for some number $T>0$

$$
\int_{0}^{T} u(t) d t \geq \rho_{0} \text { and } \int_{0}^{T} D(\beta(t))^{-1} d t \leq \tau^{*}
$$

where $u=G(\beta)$. If we define $\beta(t)=\alpha_{1}, t>T$, then $u(t) \geq 0, t>T$ and we see that the function $\beta$ gives the optimal solution for the problem in Theorem 1 when $\tau_{0}=\tau^{*}$ because $T(\beta) \geq T$. Since we established the uniqueness of the solution in Theorem 1 we have $\beta=\beta^{*}$. This completes the proof of Theorem 2 .

\section{REFERENCES}

[1]. R. Ghez and J. S. Lew, Interface kinetics and crystal growth under conditions of constant cooling rate, J. Crystal Growth 20, 273-282(1973)

[2] G. Gripenberg, On positive nonincreasing resolvents of Volterra equations, J. Differential Equations 30, 380-390(1978)

[3] G. Gripenberg, On Volterra equations with nonconvolution kernels, Report-HTKK-MAT-A118, Helsinki University of Technology, 1978

[4] G. Gripenberg, On the resolvents of nonconvolution Volterra kernels, Funkcial. Ekvac. 23, 83-85 (1980)

[5] G. Gripenberg, Asymptotic solutions of some nonlinear Volterra integral equations, SIAM J. Math. Anal. 12, 595-602(1981)

[6] A. Halanay, Optimal controls for systems with time lag, SIAM J. Control Optim. 6, 215-234 (1968)

[7] R. K. Miller, J. A. Nohel and J. S. W. Wong, Perturbations of Volterra integral equations, J. Math. Anal. Appl. 25, 676-691 (1969)

[8] H. Müller-Krumbhaar, Diffusion theory for crystal growth at arbitrary solute concentration, J. Chem. Phys. 63, 5131-5138(1975)

[9] W. E. Olmstead, Diffusion systems reacting at the boundary, Quart. Appl. Math. 38, 51-59 (1980)

[10] W. Rudin, Functional analysis, McGraw-Hill, New York, 1973 